- ORIGINAL ARTICLE Volume 9 Issue 12017

DOI: 10.21315/eimj2017.9.1.5

ARTICLE INFO

Submitted: 05-11-2016

Accepted: 16-01-2017

Online: 31-03-2017

\section{The Use of Neuro-Linguistic Programming as an Educational-Therapeutic Programme: Two Case Studies}

\author{
Meisam Savardelavar, Garry Kuan \\ Exercise and Sports Science Programme, School of Health Sciences, \\ Universiti Sains Malaysia, 16150 Kubang Kerian, Kelantan, \\ Malaysia
}

To cite this article: Savardelavar M, Kuan G. The use of neuro-linguistic programming as an educational-therapeutic programme: two case studies. Education in Medicine Journal. 2017; 9(1):49-58. https://doi.org/10.21315/ eimj2017.9.1.5

To link to this article: https://doi.org/10.21315/eimj2017.9.1.5

\begin{abstract}
Introduction: Neuro-Linguistic Programming (NLP) is continuously used as one of the psychological intervention in medicine, sport, and education settings. NLP through employing its techniques and language patterns could make certain changes in connections between neurological processes and therefore, restructure individuals' cognition and reform their behaviours. This contributes to certain advantages in educational-therapeutic sessions concerning helping athletes with emotional hardship, resulting from physical injuries. Objective: To examine a tailored intervention of the NLP model adapted for educational-therapeutic purposes to reduce competitive state anxiety of two elite basketball players after their rehabilitation programme from physical injuries. Method: This study used a case study approach, and employed the NLP model's collapsing anchoring, perceptual positions, and meta-model language patterns, to help both athletes to restructure their dysfunctional thoughts and memories. The athletes experienced anxiety related negative thoughts and felt emotionally difficult to perform in training and competition. Results: The intervention showed promising strategies in helping the athletes to reduce their competitive state anxiety. Also, the results obtained from the interview sessions, and the quantitative analysis showed that the NLP intervention had successfully helped the athletes to cope with dysfunctional thoughts and emotionally related anxiety. Conclusions: The NLP-based intervention, to some degree, helped the athletes to deal with dysfunctional thoughts of their past injury experiences, reducing their competitive state anxiety to compete again in the upcoming competition.
\end{abstract}

Keywords: Neuro-Linguistic Programming (NLP), Competitive state anxiety, Sport injury

CORRESPONDING AUTHOR Mr. Meisam Savardelavar (PhD Sports Psychology), Exercise and Sports Science Programme, School of Health Sciences, Universiti Sains Malaysia, 16150 Kubang Kerian, Kelantan, Malaysia | Email: ms15_med072@student.usm.my

\section{INTRODUCTION}

Neuro-Linguistic Programming (NLP) is a cognitive-behavioural model, operating on the nonconscious level of mind. This model operates as a therapeutic and counselling method, using the internal perceptions such as memories, emotions, and bodily sensations. NLP attempts to understand how language and nonconscious cognitions influence individuals' understandings of the internal and external events and 
the reactions towards such events $(1,2)$. For example, when a person experienced negative thoughts, even though the person could be favouring the positive behaviour; the emotions will result in sticking back to the distorted thoughts, causing the person to experienced adverse reactions (3).

Over the last decade, the NLP model has received much attention for its effectiveness in clinical (4), learning and education (5), and sports settings (6). For example, Kirenskaya et al. (7) demonstrated a significant effect employing NLP to reduce the negative emotional intensity and autonomic activity for hypnotisable participants. In addition, there has been much research supporting the applications of NLP, working with the submodalities (finer distinctions) of clients' thoughts experiencing phobias (8-9), anxiety (11-12), and post-traumatic stress disorder (13).

Recently, the application of NLP as a psychological intervention appears to be growing in certain disciplines of physiotherapy such as chronic pain, and anterior cruciate ligament rehabilitation (14-15). For example, Karunaratne (16) suggested to use the NLP model for intervention had been successfully used to eliminate the chronic pain of the participants and their perception of pain. The study also demonstrated a close relationship between the body and mind within the NLP's theory introduced by Bandler et al. (3) suggesting that, by assessing its potential in meeting the performance demands of the elite athletes, NLP could be used to investigate the emotional and psychological difficulties related to sports injuries.

According to Adirim and Cheng (17), younger athletes are more prone to sports related injuries compared to the older ones. The psychological characteristics of younger athletes usually are more impulsiveness and recklessness during competition. Thus, injuries may cause the athletes to kerb their participation in both training and competition due to their negative thoughts experienced related to re-injury and performance failure (18). In these circumstances, resistance for being involved with the possible challenges in training and competition could restrict the athletes' opportunities to learn the injuries they experienced. Therefore, it is important to evaluate the efficacy of different psychological models to identify more effective interventions, helping the athletes to overcome the psychological difficulties during, and after their recovery period.

Within the NLP model of dysfunctional thoughts (19), it was believed that the increase in anxiety is due to the mind of selfrestructuring of the dysfunctional thoughts, which mislead a person to visualise a narrow observer position on the inner process and misinterpret the thoughts (5). Thus, by applying the NLP model, it could leads the person back to visualise multiple observer positions, and to understand the meaning of such thoughts and emotions, to self-regulate the mind and body, and to correct the dysfunctional thoughts. The objective of this study was to introduce the NLP model, as an educational approach, in helping the athletes to cope with the emotional hardship, through expanding their understanding of the sport-related unpredictable events such as sports related injuries. We put forward the idea that a tailored NLP model would be beneficial for injured athletes to help them having a more effective re-entry to training and competition, getting ready to compete after their recovery period. To achieve the objective, we employed the case-study design to understand the experiences of two elite basketball players, who suffered from dysfunctional thoughts due to the right eyelid and nose injuries, respectively. Besides, the study also intended to understand the effectiveness of a tailored NLP intervention, helping injured athletes to cope with their emotional hardship during training and competition. Due to the lack of documented protocol for NLP intervention, we developed a sixsession NLP-based programme adapted 
for educational-therapeutic purposes. The core of this programme includes: (a) Clearing language of the clients, (b) Representing thoughts in mind as in both conscious and nonconscious information through five senses, (c) Changing the structure of represented thoughts working with their finer distinctions, also known as submodalities in NLP, (d) Accessing to the thoughts with opposing emotional content, (e) Pairing these thoughts and its emotional content with the challenging situation in mind, (f) Future pacing, and (g) Examining the new behaviour in reality $(1,9,21-23)$.

\section{METHODS}

\section{Participants}

Two injured male basketball players aged 16 and 17 years old, were introduced by their coach from the State's basketball club, three months after their recovery, asking for the assistance to address their psychological difficulties during training and competition.

\section{Instruments}

Demographic questionnaire. A demographic form was used to understand the participants' general backgrounds, sporting histories, and sport injury histories.

\section{Competitive State Anxiety Inventory} (CSAI-2) (24). CSAI-2 assesses the multidimensional aspects of anxiety. It has 27-items measuring three subscales, namely, cognitive state anxiety, somatic state anxiety, and self-confidence. Athletes were asked to indicate "How you feel right now" for each item on a 4-point Likert scale ranging from "Not at all" to "Very much so." Examples of the cognitive anxiety items include "I am concerned about this competition," and "I am concerned about choking under pressure." These items differ from the somatic anxiety statements such as "I feel nervous" or "I feel tense in my stomach." The self-confidence subscale includes items such as "I feel at ease," and "I'm confident
I can meet the challenge." Each of the three subscales has nine items, which are summed to get a score representing the level of intensity the athletes' feeling for each component of anxiety, and self-confidence. Studies conducted on CSAI-2 showed appropriate test-retest validity with alpha = $.80, .85$, and .88 respectively (25).

\section{Procedure}

The study received human ethics approval from the authors' institutional Human Research Ethics Committee (Code: 15040108) and followed the guidelines outlined by the Declaration of Helsinki. After the first interview session, participants were asked to complete the CSAI-2 for the first time 20 minutes before participating in a competition as the pre-test. After session four, the participants filled out the CSAI2, again, 20 minutes before taking part in the second competition as the mid-test, and finally, CSAI-2 was again completed 20 minutes before the third competition, after the sixth sessions, as the post-test. Then, a second semistructured interview session was conducted after the last session for both athletes separately to investigate their perceptions of competition and the intervention. Upon completion of the data collection, participants were provided with a debriefing form and opportunity to ask questions.

Intervention. The six NLP-based sessions were used to deliver the intervention. These sessions include "perceptual positions" and "meta-model" language pattern as the educational phase for first three sessions, then followed by "collapsing anchoring" and "meta-model" language pattern as the second three sessions of the therapeutic period. During the first three sessions, the practitioner aimed to teach participants to adopt different perspectives when observing their thoughts or encountering challenging situation during competition. They were taught to pay attention to the different interpretations they experienced during challenging events related to competition. 
Participants were educated on methods by which they could observe during competition and relates the thoughts from different observer positions, which is "I," "You," and "Others," knowing that it was an imagery process of the event. For the "collapsing anchoring" technique during the second phase of this study, practitioner aimed to guide the participants to take out the dysfunctional thought that occurred before and during training and competition. During this phase, participants were guided to recall memories of their optimal performances, and to intensifying positively related emotions, such as confidence and commitment. Thus, the participants need to learn to reject their current dysfunctional thoughts and emotional content.

Meta-model questions. This educational activity is to identify the language patterns that are believed to reflect fundamental cognitive processes of the clients during the therapeutic sessions (26). According to Grinder and Bandler (2), clients' patterns of language are good indicative of the ways a person interpret the external events and to understand their thoughts. The metamodel questions applied in the therapeutic sessions were based on the assumption that individual will understand the external events, which are not the actual events, but the representations of the events (24). We employed the meta-model language patterns to gain the necessary information needed from the two introduction sessions with the participants. Besides, we would like to get a deeper understanding of the clients' thoughts while they involved in a competition, and to reflect on the clients' thoughts, helping them to self-inform about the way they interpret the competition performance and the emotional difficulties.

Collapsing anchoring. In the collapsing anchoring technique, the memories, with the opposing emotional contents will be experienced in the client's mind, resulting in the recreation of an original emotional content of the thoughts related to the critical situation, leading to competitive state anxiety. Thus, the practitioner, first, recalls the memory containing state anxiety in the client's mind and anchors it, for example, by touching the client's knee. Next, the practitioners asked the client to remember the positive emotional contents, by providing a guide to intensify the submodalities and anchors the emotional contents. Then, the practitioner asked the client to recall the memory and then an anchoring technique was reactivated, with the negative anchor was removed and was replaced with the positive one. In this session, the dysfunctional thought related to a given situation in competition performance, and the positive emotional states of mind rooted in memories of optimal performances in the past were intensified on the submodalities. Therefore, in the future, when the client wanted to participate in a competition and had experienced dysfunctional thoughts, the client could able to reframe the thoughts automatically. In this case-study, the practitioner has to ask both athletes to imagine their future competition vividly, and whenever they could perform at the peak of their competition with emotionally related thoughts. Then, the practitioner will reactivate the positive anchor to pair its content to the thought of the future competition performance.

Perceptual positions. In NLP, perceptual positions are considered as an experiential distinction. Thus, when individuals are representing memories of the past, they pre-reflectively and perceived it from three different points of view (27). Therefore, if an athlete represents memories related to their performance from their point of view, they are in the "I" position of the observation. If they perceive information from another person's perspective like their coach, they are adopting "You" position. In addition, if an athlete is interpreting their mental representation of the past related to their performance as an independent observer, they are in "Others" position. In the NLP model, it has suggested that individuals' understanding of their mental contents could change according to the perceptual 
position they adopted (28). As opposed to the original application of perceptual positions technique, we focused more on the importance of adopting three different positions by observing, verbalising, and understandings of these thoughts through working with its' structures.

\section{RESULTS}

For confidentiality and ethical consideration, the participants in this article are referred by using pseudonyms.

\section{Case Study 1:"James"}

James is a 16-year-old male athlete, who participated with an elite basketball team. James had been competing in professional basketball tournaments for three years, but after the nose surgery (injured during a competition), he was experiencing the anxiety of taking part in the competition, and reluctant to participate in a formal competition. He had also reported that he used to spend 12 hours per week in training basketball, but because of anxiety, getting more pessimistic about other teammates and his coach's estimation of his capabilities, he was not willing to train as much as before. He was self-disappointed. He was introduced via his coach because of his noticeable recent drop in training and competition after the recovery period. In addition, it is also noted that James also experienced knee injury one year before his nose injury but did not experience emotional hardship that much after the recovery period.

Assessment. The initial James's result from CSAI-2 indicated that he was experiencing a high level of competitive state anxiety. Following the intervention, James's scores for the second and third time showing a gradual decrease in competitive state anxiety (see Figure 1).

First phase of the interview. During the first two sessions, most of the time, James's conversation hovered around the emotional challenges he was experiencing from the onset of injury, with his coach and his teammates' appraisement on his sports capabilities. James described that as the onset of his nose injury, and he was worried about the shape of his nose after surgery and also the way other peers are going to react. He said, "I don't know why I cannot have the same feeling about myself before the injury." James' initial anxiety was coupled with difficulties to interacting with his school's classmates. He commented, "you know when you have to think too much about others' thoughts during a good day; you cannot have a perfect training at evening, and that is worse if you are going to participate in a competition which may cause you to get re-injury."
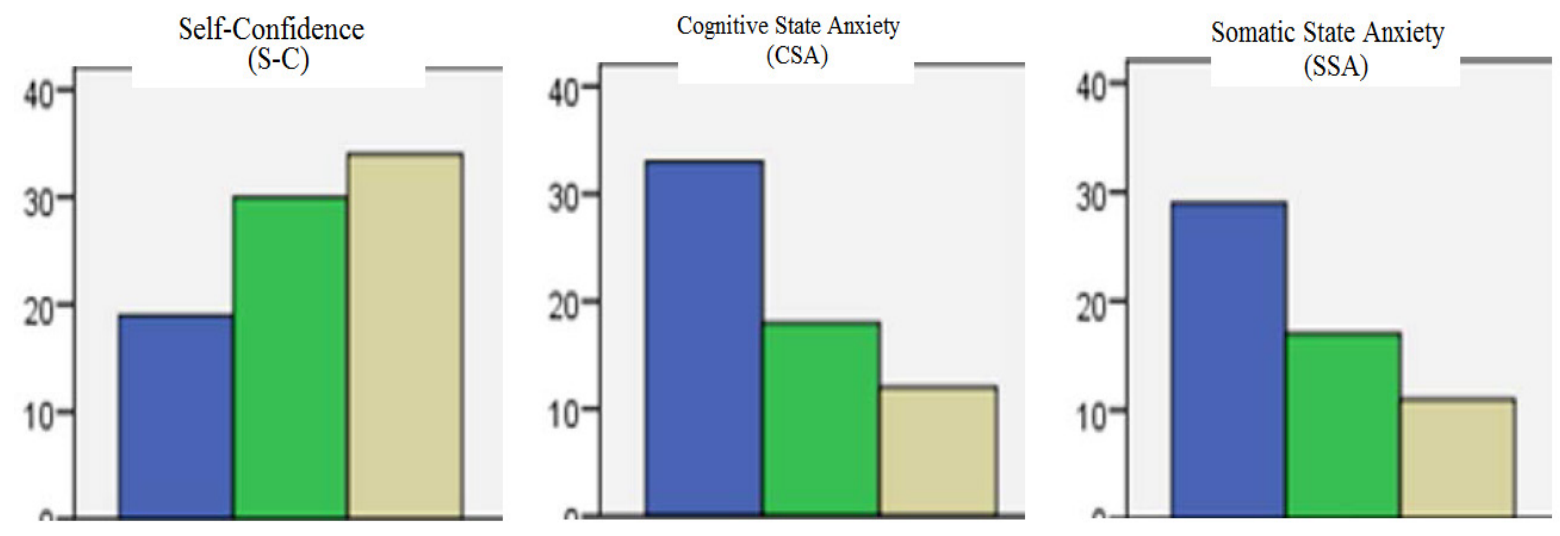

Figure 1: James's pre-mid- and post-intervention scores on Competitive State Anxiety Inventory (CSAI-2). 
"I have to make a balance between negative thoughts and staying motivated to participate in training to prepare myself for the real competition, but I have difficulties in executing my skills. My coach is helping me to regain my motivation, but it's difficult when you have a sensitive and painful nose on your face." James also showed his prediction about getting injured again, especially his nose: "I am participating in training these days, but I always think that I am going to hurt myself again, and if it happens, I will die." These statements also reflected the reasons for his high cognitive state anxiety.

Second phase of the interview. During the second phase, James commented on his mental experience and the effect of the collapsing anchoring technique on his thoughts and feelings during the sessions. From the educational part, James stated, "I'm wondered I could have several different understanding participating in the competition in my mind and fortunately, now, I'm able to choose any one of them that is more helpful. It is good, and I felt better. I've learnt that thoughts are not representing the reality of competition, but they are just my interpretations." James also identified that adopting multiple perspectives upon his thoughts and also using the critical outer event could help him to observe his thoughts without being involved himself with it. James said "When I chose the "I" observer position in my mind, everything seemed to be real, but when I adopted the "You" position, I realised that I could be more optimistic and less emotional about my basketball competition performance. Surprisingly, James commented "when I chose the "Other" perspective in my mind, I understood that I can observe most of my thoughts without being involved with them too closely, and it helped me to have a new perspective of my thoughts and feelings when I represented those thoughts on participating in the competition." $\mathrm{He}$ also stated that the collapsing anchoring technique had allowed him to reduce the negative emotional component of his negative thoughts while participating in the competition. He said "Even though I have those thoughts that used to scare me, but now when I think about it, I feel neutral, and I felt more confident. Now, I can face the new event and enjoy the experience, although I still don't know what will happen in the competition." James also stated that the NLP sessions have helped him to deal with the other part of his problems. $\mathrm{He}$ said, "I think, now, I don't have that many thoughts about how others could estimate my appearance because of the shape of my nose. I am trying to behave like before the injury, letting myself visualise what is going to happen, and I am more confident now".

\section{Case Study 2: "Jared"}

Jared is a 17-year-old male elite basketball player, who was introduced by his coach. $\mathrm{He}$ had started playing basketball since he was 12 and enjoyed playing the sport before his right eyelid was injured. Jared was playing at the State elite level of competition, spending at least 14 hours per week playing basketball. However, Jared was injured while competing in a competition, with no prior history of other major injury. When he was referred to us after his recovery, although he seldom involved with the competition, he told us that because of his terrible anxiety level before and during competition; it is hard for him to perform optimally in his game. 

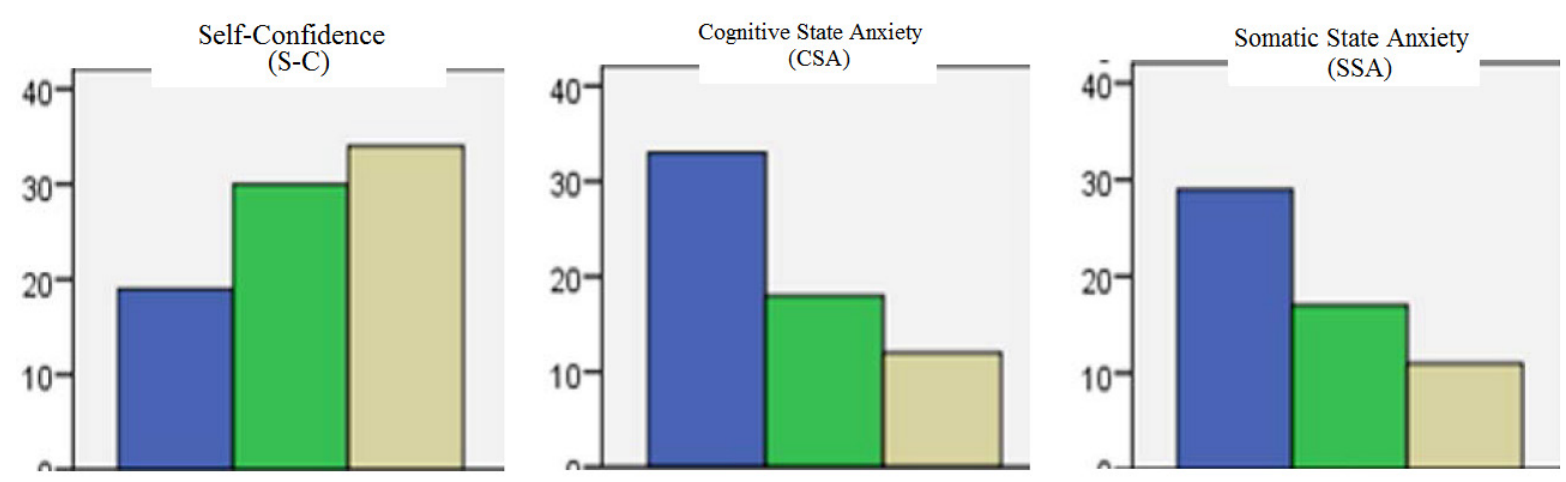

Figure 2: Jared's pre-mid- and post-intervention scores on Competitive State Anxiety Inventory (CSAI-2).

Assessment. The first CSAI-2 score demonstrated that Jared was experiencing a high level of competitive state anxiety. Following the intervention, the second and third scores showed a significant decreased in Jared's competitive state anxiety (see Figure 2).

First phase of the interview. During this phase of the interview, Jared described himself as an athlete who was facing the anxiety of reinjuring after completed his recovery course. He reported being distressed when feeling guilty and is losing his motivation to participate in any competition. He was afraid of his basketball skills getting worse and faced more critical anxiety regarding his performance capabilities. Jared also mentioned that as a result of his worries and anxiety, he has no confident to play well again during competition. However, he is asking more questions using the meta-model, showing that he is willing to cope with his psychological hardship, but was suspicious about the intervention. For example, he stated that "Is this works? If maybe I need to be more involved with the challenging situations in competition to increase my confidence faster." $\mathrm{He}$ also commented, "what if after the intervention I could not apply the skills I learnt?"

Second phase of the interview. At the end of the sessions, Jared stated that the educational part seemed to be effective for him. He commented, "the educational part helped me to enhance my understanding of the competition events, and I learnt to be responsive rather being reactive to my thoughts and outer events as well, but the collapsing anchoring did not care about my (conscious) tendency to feel negative for sometimes, and it just wanted to change my mental images replacing with positive ones which I really could not understand its philosophy behind". He also stated, "when I can see a competition events from all the "I", "You", and "Others" positions, I can see if something is going to happen to me, and for many occasions I cannot stop them, but I know I could have different feelings about what is going to happen. After all, they are thoughts and not the reality". These statements were as opposed to Jared's first phase of interview's comments where he could see his situation only from "I" observer position, feeling anxiety, and make a negative prediction about the possibility of reinjuring in competitions. We considered above contrast as a reflection of Jared's change in his approach to overcome cognitively reinjure.

\section{DISCUSSION}

The purpose of this study was to investigate the experiences of two injured elite basketball players, who faced emotional and psychological hardship after their recovery period. Next, the study also attempted to examine the effectiveness of a tailored NLPbased educational-therapeutic intervention 
in helping the two basketball players to reduce their cognitive anxiety during training and competition. The study found that first, the injured athletes captivated in their kerbed understanding of their interpretation of the current training and competition situation. Second, the tailored NLP model is useful for these athletes to adopt an observer position and to interpret their circumstances from a new perspective, to replace the dysfunctional thoughts by using the collapsing anchoring technique, and thirdly, to provide support to help the athletes facing with psychological hardship.

Both athletes experienced anxiety, negative thoughts and emotions, which had contributed to the slow progress and affected their relationship with their coaches and teammates. This study showed that the athletes preferred using the intervention strategies as educational compare to being solely therapeutic focused. This is because the educational part of the intervention gave them the ability (a) To observe their thoughts from the different point of views, and (b) To identify the thought as thoughts only, and it is not the reality. The observer positions also helped them to shift their understanding between three ("I," "You," and "Others") perspectives and had increased their knowledge about the inner processes when they faced difficulties.

Results from the findings suggested that the NLP-based educational-therapeutic intervention may help athletes to reduce the level of competitive state anxiety after the recovery period. Similarly, findings from Karunaratne (16) found similar results in adopting NLP-based intervention and suggested that NLP is an effective psychological model to cope with anxiety and psychological difficulties.

We identified two limitations from the findings. First, there is a lack of welldocumented protocol from its founders. As a result, we have to modify our own's six-sessions protocol according to all the principles of NLP. Another limitation comes from the arguments of the NLP founders regarding the effectiveness of the NLP model as a single fixed session therapy, although according to the NLP history and its effectiveness, the main part of NLP founders' arguments sound more artistic rather than actual scientific psychological intervention in the applied setting. In this current study, we followed the sixsession protocol, however, we suggested a longer period of intervention such as using the dose-response modal to identify the minimum and maximum response rates.

These case studies provided an important opportunity to advance the understanding of NLP's mechanisms for reducing performance anxiety and restructuring negative thoughts. However, more research on these topics needs to be undertaken before the association between the effectiveness of NLP-based interventions and performance anxiety is fully understood. Thus, we recommend that future research to continue investigating using the singlecase study design to explore the effectiveness of NLP to support the needs of athletes with difficulties in reentry to competitions after their recovery course. Considering the complicated nature of NLP approach on human cognition, there is abundant room for further investigation in determining the effectiveness of NLP in another field of human performance like surgery in which performance anxiety, the ability of selfobservation could play a crucial role. This helps to gain more understanding of NLP's underlying mechanisms that could help the individuals to moderate their emotiondriven performance. Also, we suggested that as long as making considerable changes in a way NLP techniques are employed by academic researchers could be done to meets the demands of psychological hardship normally individuals face during their performance.

\section{CONCLUSION}

The study investigates the effectiveness of a tailored NLP-based intervention to meet the needs of two elite basketball 
players, and to reduce their competitive state anxiety after the injury recovery period. The educational section of the intervention helped the athletes to adopt the multiple points of view over competition performance during challenging situations to broaden their understanding of the conditions. This study, also, attempted to take out the emotional components of dysfunctional thoughts, which occurred before and during competition. In general, this study demonstrated that injured athletes experienced many negative thoughts, often worried about their physical appearance. Thus, the six-session educationaltherapeutic NLP-based intervention demonstrated that it is an effective tool for helping the athletes after their recovery course to adopt an observer positions over their thoughts and emotions, related to the challenging situations and to restructure their dysfunctional thoughts. Collectively, the NLP-based intervention appeared to provide injured athletes with the opportunity to reduce their anxiety and to perform better in their upcoming competitions.

\section{ACKNOWLEDGEMENTS}

The present study was supported by the Research University's Individual Grant (USM-RUI) from Universiti Sains Malaysia (1001/PPSP/812149).

\section{REFERENCES}

1. Bandler R, Grinder J. The structure of magic: I. A book about language and therapy (Vol. 1). Palo Alto, CA: Science and Behavior Books; 1975.

2. Grinder J, Bandler R. The structure of magic II: A book about language and therapy (Vol. 2). Palo Alto, CA: Science and Behavior Books; 1976.

3. Bandler R, Grinder J, Andreas S. Neurolinguistic programming ${ }^{\mathrm{TM}}$ and the transformation of meaning. Utah: Real People; 1982.
4. Wake L, Gray R, Bourke F. The clinical effectiveness of neuro-linguistic programming: a critical appraisal. Oxon: Routledge; 2013.

5. Fakehy M, Haggag M. The effectiveness of a training program using neuro-linguistic programming (NLP) to reduce test anxiety in consideration of biological feedback. Int J Behav Res Psychol. 2016;4(1):173-7. https:// doi.org/10.19070/2332-3000-1600031

6. Saunders DE. A psycho-educational programme for cricket players using neurolinguistic programming (Dissertation). South Africa: University of Stellenbosch; 2009.

7. Kirenskaya AV, Novototsky-Vlasov VY, Chistyakov AN, Zvonikov VM. The relationship between hypnotizability, internal imagery, and efficiency of neuro-linguistic programming. Int J Clin Exp Hypn. 2011; 59(2):225-41. https://doi.org/10.1080/00207 144.2011 .546223

8. Allen KL. An investigation of the effectiveness of neuro-linguistic programming procedures in treating snake phobics (Dissertation). Kansas City: University of Missouri; 1982.

9. Liberman $\mathrm{MB}$. The treatment of simple phobias with neuro-linguistic programming techniques (Dissertation). St. Louis: St. Louis University; 1984.

10. Einspruch, EL, Forman BD. Neurolinguistic programming in the treatment of phobias. Psychotherapy in Private Practice. 1988;6(1):91-100.

11. Hale RL. The effects of neuro-linguistic programming (NLP) on public speaking anxiety and incompetence (Dissertation). Parkville: Parke University; 1986.

12. Ferguson DM. The effect of two audiotaped neuro-linguistic programming phobia treatments on public speaking anxiety (Dissertation). Tennessee: University of Tennessee; 1988. 
13. Al-Harbi KS. Treatment-resistant depression: therapeutic trends, challenges, and future directions. Patient Prefer Adherence [Internet]. 2012 (cited 2014 Oct 14);6:369-88. Available from: http:// www.ncbi.nlm.nih.gov/p mc/articles/ PMC3363299. https://doi.org/10.2147/PPA. S29716

14. Alexanders J, Anderson A, Henderson S. Musculoskeletal physiotherapists' use of psychological interventions: a systematic review of therapists' perceptions and practice. Physiotherapy. 2015; 101(2):95-102. https://doi.org/10.1016/j. physio.2014.03.008

15. Bolstad R, Prochazka L. NLP and relief of chronic pain. Anchor Point Magazine. 2003; $17: 1$.

16. Karunaratne, M. Neuro-linguistic programming and application in treatment of phobias. Complement Ther Clin Pract. 2010;16(4):203-7. https://doi.org/10.1016/j. ctcp. 2010.02.003

17. Adirim TA, Cheng TL. Overview of injuries in the young athlete. Sports Med. 2003;33(1):75-81. https://doi. org/10.2165/00007256-200333010-00006

18. Mahoney J, Hanrahan SJ. A brief educational intervention using acceptance and commitment therapy: four injured athletes' experiences. J Clin Sport Psychol. 2011;5(3):252. https://doi.org/10.1123/ jcsp.5.3.252

19. Harman RL, O’Neill CH. Neuro linguistic programming for counselors. Pers Guid J. 1981;59(7):449-53. https://doi. org/10.1002/j.2164-4918.1981.tb00593.x
20. Grinder J, De Lozier J, Bandler R. Patterns of the hypnotic techniques of Milton $\mathrm{H}$. Erickson, MD Vol. II. Cupertino, CA: Meta Publications; 1977.

21. Bandler R, Grinder J. Frogs into princes: neuro-linguistic programming. Moab, Utah: Real People Press; 1979.

22. Hall M. Communication magic. Wales: Crown House Publications; 2001.

23. Grinder J, De Lozier J, Bandler R. Patterns of the hypnotic techniques of Milton $\mathrm{H}$. Erickson, MD Vol. II. Cupertino, CA: Meta Publications; 1977.

24. Martens R, Vealey RS, Burton D. Competitive anxiety in sport. NY: Human kinetics; 1990.

25. Lane AM, Sewell DF, Terry PC, Bartram D, Nesti MS. Confirmatory factor analysis of the competitive state anxiety inventory-2. J Sports Sci. 1999;17(6):505-12. https://doi. org/10.1080/026404199365812

26. Johnson MI, Hudson M. Generalizing, deleting and distorting information about the experience and communication of chronic pain. Pain Manag. 2016;6(5):41114. https://doi.org/10.2217/pmt-2016-0028

27. Dilts R, DeLozier J. Encyclopedia of systemic NLP and NLP new coding. Scotts Valley, CA: NLP University Press; 2000, 849-55.

28. Andreas C, Andreas T. Aligning perceptual positions: A new distinction in NLP. J Conscious Stud. 2009;16(10-1):217-30. 